\title{
Construção de habilidades em Segurança Alimentar e Nutricional com titulares de direito do Programa Bolsa Família ${ }^{1}$
}

\author{
Estelamaris Tronco Monego², Karine Anusca Martins², Maria Janaína Cavalcante Nunes ${ }^{3}$, \\ Raquel Andrade Cardoso Santiago ${ }^{2}$, Marcia Helena Sacchi Correa ${ }^{2}$, Lucilene Maria de Sousa ${ }^{2}$, \\ Veruska Prado Alexandre ${ }^{2}$, Maria Raquel Hidalgo Campos ${ }^{2}$
}

O direito humano à alimentação adequada (DHAA) é reconhecido como o acesso regular e permanente a alimentos, sem qualquer tipo de restrição qualitativa ou quantitativa. Tem como premissa a alimentação segura, adequada e saudável. Esse artigo relata a experiência de um projeto que promoveu atividades de Segurança Alimentar e Nutricional (SAN) e DHAA, com trabalhadores e titulares de direito envolvidos com o Programa Bolsa Família (PBF). Participaram deste projeto dezenove municípios do estado de Goiás, incluindo a capital. Foram realizadas ações de formação com profissionais da saúde, educação e assistência social tendo como enfoque o PBF. Os profissionais vinculados ao Sistema Único de Assistência Social (SUAS) formularam planos de ação municipais. Os titulares de direito do PBF participaram de formações voltadas para a SAN e DHAA, incluindo roda de conversa sobre os direitos humanos e oficina de culinária. A experiência claramente demonstrou como o projeto incentivou os titulares de direito e equipe do PBF a produzir conhecimento e novas práticas em suas cidades e vida. A equipe, os grupos profissionais envolvidos e o público participante, consideram que esta ação foi importante na execução e na promoção de uma alimentação adequada e saudável, conforme determina o PBF.

Palavras-chave: Segurança alimentar e nutricional, Vulnerabilidade social, Programas governamentais, Direitos humanos..

\section{Construction of skills on Food Safety and Nutrition with right holders of "Bolsa Familia" Program}

The Human Right to Adequate Food (HRAF) is recognized as having regular and permanent access to food without any qualitative or quantitative restrictions. Its premise is safe, adequate, and healthy food. This article describes the experience of the project that promoted Food and Nutrition Security (FNS) and HRAF alongside citizens entitled to the Bolsa Familia Program (BFP) and its staff. Nineteen cities from the state of Goiás were part of this project, including the capital. Training sessions were conducted for health, education, and social professionals that worked with BFP. The staff from Universal System of Social Assistance formulated local action plans. The project staff trained and informed the citizens entitled to BFP through discussions, conversation circles, and cooking workshops. The experience clearly demonstrates how the project was able to equip citizens and staff to produce relevant knowledge and new practices both in their cities and their lives. The involved staff, working groups, and citizens considered this action as important to healthy and adequate food execution and promotion, according to FBP.

Key-words: Food and nutrition security, Social vulnerability, Government programs, Human rights.

\footnotetext{
${ }^{1}$ Este artigo é produto de experiência vivenciada em atividade de parceria da Faculdade de Nutrição e a Secretaria de Cidadania e Trabalho de Goiás denominada "Projeto de Educação Alimentar e Nutricional e de Difusão do Direito a uma Alimentação Adequada" financiado pelo Ministério do Desenvolvimento Social e Combate à Fome”. ${ }^{2}$ Faculdade de Nutrição. Universidade Federal de Goiás (UFG). Rua 227, Quadra 68, s/no - Setor Leste Universitário. CEP: 74.605-080. Goiânia - GO.

${ }^{3}$ Coordenação de Vigilância Nutricional. Superintendência de Vigilância em Saúde. Secretaria de Estado de Saúde de Goiás. 


\section{INTRODUÇÃO}

O Brasil tem se destacado internacionalmente quanto ao desenvolvimento de um escopo de políticas públicas promotoras da Segurança Alimentar e Nutricional (SAN) e do Direito Humano à Alimentação (DHA) ${ }^{[1,2]}$. Este conjunto de ações públicas, associadas a outras medidas de ordem econômica como o aumento real do salário mínimo, e também sociais como o acesso a rede de educação e saúde, tem levado a importantes resultados, como a redução da desnutrição e mortalidade infantil ${ }^{[3]}$, além da redução expressiva do número de pessoas em situação de fome no país [4].

Este cenário também pode ser resultado da forma como a sociedade civil tem se inserido nos processos de participação social, como os conselhos de SAN, Saúde, Educação, Alimentação Escolar, entre outros. Embora se tenha essa avaliação positiva, vê-se ainda a necessidade de ampliação desta participação, integrando novos atores e promovendo o processo de construção e acompanhamento de políticas públicas, assim como de situação de violação de direitos, entre esses, o Direito Humano à Alimentação Adequada (DHAA) no cotidiano.

O DHAA origina-se no Pacto Internacional dos Direitos Econômicos, Sociais e Culturais (PIDESC), que o reconhece atrelado a um padrão de vida adequado, incluindo a alimentação adequada e saudável, fundamentalmente, ao seu acesso regular, permanente, sem qualquer tipo de restrição, em quantidade e qualidade suficientes e apropriadas às diferentes necessidades e culturas, tendo como base práticas alimentares individuais e coletivas promotoras de saúde ${ }^{[5,2]}$.

A promoção da saúde, nessa perspectiva, apresenta-se como um conjunto de estratégias com vistas a fortalecer o diálogo, a articulação e a cooperação intra e inter setorial para formação da Rede de Atenção à Saúde (RAS). Assim, faz-se necessário articular ações de proteção social para uma melhor qualidade de vida da população, com ampla participação e controle social, para que todos sejam partícipes na proteção e cuidado com a vida $[6,7]$.

Neste contexto, o DHAA, nas dimensões "direito de estar livre da fome e da má nutrição" e "direito à alimentação adequada"[5], baseia-se em práticas de significado social e cultural, onde os alimentos devem ser considerados em sua totalidade, com respeito às suas características sensoriais próprias para suprir as necessidades individuais e coletivas ${ }^{[6]}$.

Ampliando o olhar, são necessárias estratégias políticas que assegurem o DHAA como medida indispensável para a saúde da população. Nesse escopo, situa-se o Programa Bolsa Família (PBF) ${ }^{[8]}$, cujo objetivo principal é complementar a renda de famílias em situação de vulnerabilidade, dando condições para sua emancipação. Seu alcance é possível por meio da articulação de três dimensões: a promoção do alívio imediato da pobreza, por meio da transferência direta de renda; o reforço ao exercício de direitos sociais básicos nas áreas da Saúde, da Educação e da Assistência Social e, a interface com programas complementares de geração de trabalho e renda, alfabetização de adultos, de fornecimento de registro civil e demais documentos ${ }^{[?]}$.

Reconhece-se que a condição de pobreza exerce impacto direto no perfil de saúde e contribui para reforçar a vulnerabilidade social, fortalecendo o ciclo da pobreza. Os programas de transferência de renda com condicionalidades de saúde influenciam positivamente no acesso aos serviços de saúde, por possibilitar, além disso, a eliminação e/ou minimização da pobreza, com vistas a reduzir a fome, condição relevante no processo de promoção, proteção e recuperação da saúde ${ }^{[10]}$.

Neste contexto, o objetivo do presente artigo é relatar a experiência da formação em SAN e DHAA realizada junto a um grupo de titulares de direito do Programa Bolsa Família e a construção de planos de ação municipais junto a profissionais vinculados ao Sistema Único de Atenção Social.

\section{METODOLOGIA}

Trata-se de um relato de experiência sobre ações desenvolvidas no projeto de extensão denominado "Projeto de Educação Alimentar e Nutricional e de Difusão do Direito a uma Alimentação Adequada". Este foi planejado e executado em parceria pela Faculdade de Nutrição da Universidade Federal de Goiás e a Secretaria de Cidadania e Trabalho (SECT) de Goiás, no período de fevereiro a outubro de 2010. A ação teve apoio financeiro do Ministério do Desenvolvimento Social e Combate à Fome e apoio logístico das prefeituras dos municípios participantes. 
A área de abrangência do projeto incluiu os dezenove municípios que compõem a região metropolitana, além de Goiânia, capital do estado de Goiás. Neste território, a SECT de Goiás identificou o cadastro de 2.400 famílias no PBF nos municípios envolvidos. Diante do número de titulares de direito do programa, estimou-se um máximo de 60 participantes por município (Etapas 1 e 2) e 25 multiplicadores (profissionais da saúde, educação e assistência social) por município (Etapa 3). Para o seminário final foi estimada uma representação de 300 destes participantes das etapas anteriores.

Consideraram-se como critérios de inclusão ser município integrante da região metropolitana da capital e o participante ser titular do PBF (Etapas $1 \mathrm{e}$ 2), profissionais de saúde, educação e assistência social recrutados como multiplicadores (Etapa 3), além do interesse do munícipio na adesão ao projeto, acordado previamente.

O desenvolvimento do projeto envolveu atividades teórico-práticas realizadas em três etapas. A primeira e segunda etapas tiveram como públicoalvo os titulares de direito do PBF, enquanto a terceira etapa, os profissionais vinculados à saúde, educação e assistência social do município. $O$ processo educativo participativo foi fundamentado na base teórica composta pelo DHAA; a SAN e a Promoção da Alimentação Adequada e Saudável $[5,6,11,12]$.

A Etapa 1 teve por objetivos promover uma reflexão sobre os direitos humanos e (re)conhecer a alimentação como direito humano e a si mesmo como sujeito titular de direitos. Nesta etapa, realizou-se a formação dos representantes das famílias cadastradas e ativas do PBF, com duração total de doze horas.

A estratégia de trabalho envolveu momentos sequenciais, onde inicialmente era repassado um prato de papel onde cada um deveria registrar, em palavras escritas em uma tira de papel ("Dinâmica do Prato"), as expectativas em relação ao curso. Posteriormente, os participantes eram divididos em grupos para discutir suas percepções e conhecimentos sobre direitos humanos e DHAA, com apresentação de situações práticas do cotidiano, possibilitando a reflexão e discussão sobre essa temática. $\mathrm{O}$ produto dessa discussão se traduzia em cartazes com construção de conceitos sobre os temas geradores, desenhos/recortes de figuras que remetiam a situações de ausência de direitos, culminando com a exposição do filme "Peraí, é nosso direito"[13], acompanhada de questões orientadoras.

O conceito de SAN era articulado com provérbios populares, músicas e figuras, finalizando com a apresentação de cada grupo sobre suas percepções e conceituação sobre a temática. Foi apresentado e discutido o cenário sobre a situação de saúde e nutrição no Brasil e em Goiás. Um roteiro elaborado em multimídia trazia ao grupo "questões que eu sempre quis saber e não tinha a quem perguntar" que envolvia aspectos relacionados ao tema e o conhecimento popular com a inserção de alguns adágios populares: 'saco vazio não para em pé;; 'onde come UM comem DOIS'; 'nem só de pão vive o Homem' e 'o que não mata engorda' instrumentalizando-se os participantes para sua autonomia na utilização racional dos recursos do PBF.

Ao final, agendava-se a Etapa 2, destinada a resgatar de forma prática os conteúdos abordados na Etapa anterior. Esta etapa ocorria em único turno, incluindo um momento teórico seguido da preparação e degustação de quatro receitas de baixo custo financeiro, com alimentos regionais e da época (suco da horta, arroz verdinho, miscelânea de legumes e curau de abóbora), previamente testadas no Laboratório de Dietética da Faculdade de Nutrição da Universidade Federal de Goiás ${ }^{[14]}$.

Esta etapa abordava, de forma mais laborativa, cuidados que envolviam a SAN (compras versus orçamento familiar; higiene pessoal e dos alimentos; aspectos sensoriais e nutricionais; direitos e deveres do consumidor), seguida de atividade onde eram elaboradas preparações culinárias saudáveis e de baixo custo, buscando incentivar $O$ aproveitamento integral dos alimentos, o uso moderado de sal, óleo e açúcar, bem como, as boas práticas de manipulação. Como apoio didático elaborou-se uma cartilha, que sintetizava os conteúdos abordados na primeira e segunda etapa [15].

A Etapa 3 teve como público-alvo os profissionais responsáveis pela execução do programa no município, dos setores da educação, saúde e assistência social. A dinâmica contemplou a discussão sobre SAN e DHAA, por meio do compartilhamento de experiências, em atividade que visava compor uma árvore de problemas, definição de prioridades e posterior elaboração dos Planos de Ação municipais. Esta Etapa teve uma duração de oito horas/aula que foi complementada por outras 
sete horas/aula na modalidade de ensino à distância, onde um professor tutor orientava as ações dos grupos de cada município. A finalidade era a elaboração e posterior execução de um Plano de Ação municipal com vistas à (re)organização das ações do PBF.

Ao final das três etapas, realizou-se um seminário de avaliação envolvendo os multiplicadores - representantes dos municípios e instituições parceiras do projeto que executavam suas ações nos territórios habitados pelo público inscrito no PBF. O objetivo foi compartilhar experiências, discutir os Planos de Ação municipais e avaliar a implementação do projeto.

O projeto de extensão foi aprovado pelos Conselhos Estaduais de Assistência Social e de Segurança Alimentar e cadastrado no Sistema de Acompanhamento de Projetos, da Pró-reitoria de Extensão e Cultura da Universidade Federal de Goiás e aprovado pelo Comitê de Ética em Pesquisa Dr. Henrique Santillo, da Secretaria de Estado da Saúde de Goiás.

\section{RESULTADOS E DISCUSSÃO}

Participaram do estudo 19 municípios, sendo 18 da região metropolitana (Abadia de Goiás, Aparecida de Goiânia, Bela Vista, Bonfinópolis, Brazabrantes, Caldazinha, Caturaí, Goianápolis, Goianira, Guapó, Hidrolândia, Inhumas, Nerópolis, Nova Veneza, Santo Antônio, Senador Canedo, Terezópolis e Trindade) e a capital Goiânia, alcançando cerca de 2.000 pessoas, das quais 1600 nas Etapas 1/2 e 200 profissionais na Etapa 3 e 200 representantes no Seminário Final.

O projeto foi segmentado em etapas para alcançar os objetivos propostos, buscando-se a transversalidade dos temas geradores: DHAA; SAN e promoção da alimentação adequada e saudável (PAAS), em um conjunto de atividades teóricopráticas.

Na Etapa 1, como se tratava de um processo de construção coletiva de conceitos a partir das vivências, num primeiro momento utilizou-se a 'dinâmica do prato', quando os participantes deveriam escrever em um papel seu nome, origem e o que traziam para encher o prato no início do curso. Na sequência, o facilitador fazia a leitura e discussão das expectativas dos participantes, e em seguida relatava-se a importância do projeto no contexto do DHAA e SAN. Esta estratégia possibilitou aos participantes expressarem suas expectativas sobre o curso e teve como respostas mais frequentes a curiosidade, a vontade de aprender algo novo ou ainda de melhorar os conhecimentos sobre nutrição e saúde.

"Trago minha vontade de aprender" [Bonfinópolis]

"Eu vim porque nunca é tarde para aprender" [Caldazinha]

Esta dinâmica atuava como atividade quebragelo e oportunizava uma maior integração e aproximação entre os participantes e a equipe executora, ao mesmo tempo em que destacava a importância da partilha de conhecimentos do grupo. Além disto, contribuía para romper os desafios da nutrição no contexto da promoção de saúde, na medida que possibilitava integrar os diferentes olhares e estabelecer um diálogo participativo, construído a partir dos conhecimentos científico e popular ${ }^{[16,17]}$.

Posteriormente era realizada uma discussão do DHAA, com o objetivo de (re)conhecer a alimentação como direito humano e a si mesmo como um sujeito titular de direito. Em grupos eram discutidas as seguintes questões: o que é direito humano? $\mathrm{O}$ que você reconhece como direito seu (dos seres humanos)? Posteriormente, as respostas eram anotadas em painéis e apresentadas, cabendo ao facilitador fazer uma síntese dos direitos humanos, enfatizando o DHAA.

No decorrer da conversa, o PBF era incluído com o objetivo de discutir seu papel na garantia da oferta de alimentos e do DHAA. Essa partilha de opiniões encaminhava para a exposição do filme "Peraí, é nosso direito!", que possibilitou a reflexão sobre a indivisibilidade dos direitos humanos; a força da mobilização comunitária e o DHAA. As seguintes questões norteadoras foram utilizadas: a alimentação é um direito humano? Como vocês gostariam que fosse sua alimentação? Como o PBF pode servir a este propósito? A partir destas reflexões, ocorria a construção coletiva do conceito de alimentação adequada e saudável. Para subsidiar esta atividade utilizou-se um conjunto de figuras/imagens de pessoas com excesso de peso, desnutrição, coletando lixo e alimentos comercializados na rua.

A discussão do PBF possibilitava evidenciar seu papel como instrumento de garantia da oferta de alimentos e do DHAA. Este reconhecimento era 
reiterado no debate sobre as impressões causadas pelo filme, o que possibilitou a reflexão sobre a indivisibilidade dos direitos humanos; a força da mobilização comunitária e o DHAA. As questões norteadoras possibilitaram novas reflexões, que culminaram com a elaboração do conceito de alimentação adequada e saudável.

Neste contexto, trabalhava-se com informações sobre o estado nutricional e sobre as causas de adoecimento e mortes no Brasil e em Goiás, estimulando-se uma reflexão sobre o tema: estas pessoas têm o DHAA violado (não cumprido)? $\mathrm{O}$ resultado dessas discussões encaminhava para a construção do conceito de SAN, com a inserção de alguns adágios populares: "saco vazio não para em pé"; "onde come UM comem DOIS"; "em só de pão vive o homem" e "o que não mata engorda".

Ressalta-se que o não envolvimento do gestor do PBF; desinteresse do município em participar da atividade; falta de uma divulgação eficiente a fim de que o público-alvo comparecesse à atividade agendada previamente, tornaram-se um gargalo na execução dessa etapa, cujo conteúdo teórico visava instrumentalizar os participantes para sua autonomia na utilização racional dos recursos do PBF. Porém, oportunizaram vivências quanto às dificuldades que ocorrem quando no desenvolvimento de ações que envolvem outros segmentos, nos quais os interesses políticos terminam por interferir na participação popular. Por esta razão, foi necessário lançar mão de estratégias alternativas para garantir a realização das atividades. Assim, em alguns municípios, quando não houve o preenchimento das vagas, estas foram disponibilizadas para outras pessoas, não beneficiárias do PBF, geralmente profissionais da área da saúde ou da educação.

Importante considerar a relevância do controle social, que pode provocar reações contraditórias, já que interfere em ações historicamente exercidas pelo Estado sobre a sociedade durante muitos anos. O Estado ao transferir poderes ao cidadão, define uma carga de participação da sociedade nas decisões sobre as políticas públicas, ressaltando que estes responderão não apenas aos interesses pessoais, como também dos gestores, numa construção que sai do individual e se transforma em coletiva ${ }^{[18]}$.

Ao final da atividade, uma nova rodada da "Dinâmica do Prato" visava discutir com o grupo quais as informações obtidas naquela atividade tinham agregado conhecimentos úteis para a sua vida, por meio da seguinte questão: "eu cheguei aqui com expectativas e dúvidas e estou levando para casa um prato cheio de ... ".

A Etapa 2, agendada com o grupo na sequência da anterior, visava executar atividade capaz de resgatar de forma prática os conceitos trabalhados naquela ocasião. A parte inicial incluiu aspectos relativos à aquisição e armazenamento dos alimentos, leitura e discussão das receitas, seus substitutos e valor nutricional. Após a exposição dialogada, dava-se início à parte prática, na qual eram preparadas quatro receitas: suco da horta (suco de maracujá com couve), arroz verdinho (arroz com talos de couve e casca de abóbora kabutiá), miscelânea de legumes (torta recheada com cenoura, chuchu com casca, milho e tomate) e curau de abóbora, sendo demonstradas todas as etapas, passo-a-passo, seguidas de degustação. Para viabilizar a produção das preparações no tempo agendado, cabia a uma auxiliar de cozinha previamente treinada realizar o pré-preparo das receitas que necessitavam de um maior tempo de execução, concomitante à parte teórica. $\mathrm{Na}$ escolha das preparações foram considerados os hábitos regionais, o uso racional dos alimentos, custo, viabilidade de execução de receita, aspectos nutricionais e sensoriais, em receitas previamente testadas no Laboratório de Dietética da Faculdade de Nutrição da Universidade Federal de Goiás ${ }^{[14]}$.

A discussão sobre as boas práticas na manipulação de alimentos estimularam os participantes a rever suas práticas cotidianas e a refletir sobre a importância desses procedimentos na rotina doméstica. Já as atividades práticas possibilitaram o conhecimento de uma culinária saudável e viável por meio da discussão e produção de receitas. Esta ação permitiu estimular a criatividade e despertar nos participantes a necessidade de avaliar e discutir suas práticas domésticas no preparo dos alimentos, bem como propor soluções que contribuíssem para melhorar o valor nutritivo, o aspecto sensorial e a redução de desperdício. A fim de aumentar a acessibilidade dos participantes ao receituário, os ingredientes empregados foram padronizados em medidas caseiras (xícaras e colheres). Além disto, as receitas foram executadas passo-a-passo, com grande detalhe quanto às técnicas de preparo. Após a preparação das receitas era realizada a degustação por todos os participantes e o instrutor realizava a síntese do conteúdo, promovendo-se a avaliação da atividade pelos participantes. 
Em geral, as preparações foram avaliadas positivamente, com relato de que seriam incluídas no cardápio da família, em função do custo compatível com o orçamento, da facilidade de preparo, do valor nutricional e do sabor. Destaca-se que estes relatos apontavam a predisposição dos participantes para modificarem suas práticas alimentares em busca de uma alimentação mais saudável e com aproveitamento racional dos alimentos, seguindo as boas práticas de manipulação de alimentos. Isto permite supor que foi possível discutir o alimento e a alimentação de forma contextualizada aos aspectos social e cultural, com foco nos alimentos e não apenas em nutrientes ${ }^{[1,12]}$ :

"Eu consumia 6 a 8 L de óleo por mês e agora só uso 1,5 L” [Nerópolis]

"Nunca mais vou lavar os alimentos (verduras e legumes) com água e sabão" [Bela Vista]

"Uma palestra pode ser muito proveitosa para nossa vida ... basta saber usar "[Goianápolis]

A Etapa 3 envolveu os profissionais da Estratégia Saúde da Família (ESF), da educação e assistência social em uma atividade de formação de multiplicadores nos temas geradores, em cada um dos municípios participantes. Nesta etapa, o foco era a capacitação para trabalhadores que executam suas atividades em territórios do PBF (promoção social, saúde, educação) e na elaboração de um Plano de Ação. Realizou-se um encontro presencial com duração de oito horas/aula, além de sete horas/aula na modalidade de ensino à distância. Como estratégia de trabalho, iniciava-se com uma parte teórica que encaminhava à discussão e construção dos conceitos de DHAA e SAN, a partir dos quais se realizava uma atividade em pequenos grupos, cuja finalidade era compor uma árvore de problemas, que na sequência definia uma prioridade e elaborava um Plano de Ação.

"Vou falar para vocês de dentro do men coração: en gosto muito do que faço...” [Goiânia]

"A gente tem que ter mais amor pelo próximo, cuidar mais, ajudar mais e 'correr atrás' ..." [Caldazinha]

Cabia aos grupos elaborar um roteiro norteador que incluía a identificação dos problemas e as ações que têm sido feitas para sua resolução; a identificação da situação como vivem, adoecem e morrem as pessoas do local; quais dificuldades, potencialidades e necessidades identificam neste contexto, cujas respostas eram obtidas em uma atividade de campo. A tarefa de cada um dos grupos era responder estas perguntas posteriormente in loco, elaborar uma proposta com ações de intervenção e enviar para correção pelo facilitador. Dentre os problemas identificados listaram-se a baixa motivação da clientela; a falta de comunicação dos gestores do PBF (educação, saúde e assistência social) na condução das ações e programas relacionados à saúde e a (des)articulação saúde e promoção social na gestão do PBF.

Após a execução do Plano de Ação, ocorreu um Seminário para avaliação em Goiânia, que contou com a participação de gestores e lideranças dos municípios que aderiram à parceria nas etapas anteriores, com vistas a discutir a eficácia da estratégia e os resultados, além de propor medidas para continuidade das ações. O objetivo foi reunir representantes de todos os municípios participantes para discussão dos limites e possibilidades na execução do Plano de Ação elaborado para uma troca de experiências. Duas experiências consideradas bem sucedidas a partir de um monitoramento telefônico, foram apresentadas ao grupo.

A avaliação evidenciou inúmeros aspectos positivos, com destaque para a escolha da metodologia e dos temas abordados, que contribuiu para que os participantes fizessem a análise crítica acerca do contexto social em que estavam inseridos, conhecessem o fenômeno da transição nutricional no nosso país, e o cenário atual onde se convive com dois extremos da má nutrição - carências nutricionais e obesidade. Desta forma, contemplaram-se os campos de atuação de promoção da saúde, por meio da articulação dos saberes técnicos e populares e da capacitação dos indivíduos, uma vez que estes eram estimulados a discutir os problemas e buscar soluções ou formas de minimizá-los [16,17].

Os programas de transferência de renda condicionada, como o PBF, contribuem de forma mais efetiva para a melhoria do estado nutricional quando associada a outros tipos de intervenção que a médio e longo prazos impactam positivamente a capacidade das famílias na utilização da renda [18,19]. A garantia da SAN requer adoção de ações não isoladas e não somente direcionadas aos indivíduos pobres ou famintos, mas sim de outras medidas que impactam outras dimensões como a disponibilidade/produção de alimentos, garantia da qualidade sanitária e nutricional dos alimentos, dentre outras ${ }^{[20,21]}$. 
$\mathrm{Na}$ estruturação de ações de alimentação, nutrição e saúde, coexistem diversos desafios para sua consolidação, em caráter universal. Dentre, eles destacam-se o difícil monitoramento das condicionalidades de saúde das famílias titulares de direito do PBF, explicitando assim a necessidade de expansão e consolidação da Vigilância Alimentar e Nutricional, para garantir a avaliação periódica do estado nutricional e o consumo alimentar da população, nas unidades de saúde, bem como o fortalecimento de ações de formação junto aos titulares e profissionais envolvidos com a execução do Programa[21,22].

\section{CONCLUSÕES}

Este projeto representa um avanço enquanto estratégia participativa, cujo objeto de trabalho era uma intervenção em política governamental. Quanto aos profissionais envolvidos na ação, ressalta-se a motivação e necessidade das equipes em rever as estratégias de trabalho junto à comunidade, evidenciadas pelos Planos de Ação municipais elaborados. O projeto permitiu vislumbrar novas possibilidades de articulação do tripé Assistência Social, Saúde e Educação. Evidencia-se a importância das parcerias locais; do correto registro dos dados (retroalimentação do processo); da Educação Permanente em saúde e da indispensável fiscalização na seleção dos beneficiários.

Quanto aos titulares de direito do PBF, foi possível evidenciar que a informação tem uma ressonância forte quanto à mudança de práticas, o que sinaliza a importância de que medidas de interlocução mais horizontais devam ser efetivamente aplicadas.

Por fim, considera-se como ganho adicional a (re)descoberta do estudo na necessária renovação do conhecimento por parte das equipes locais. É possível afirmar que o mesmo atingiu seus objetivos, sendo bastante claro que a educação permanente, em todos os níveis, é necessária para qualificar as ações na saúde, educação e assistência social. E que esta qualificação pode ser utilizada pelos participantes de várias formas, mas são indispensáveis o vínculo, a responsabilidade e o compromisso, pilares filosóficos das práticas na atenção primária à saúde.

\section{REFERÊNCIAS}

\section{REFERÊNCIAS BIBLIOGRÁFICAS}

[1] De Schutter O. (United Nations General Assembly). Final report: the transformative potential of the right to food [Internet]. New York: UN General Assembly; 2014 [Acesso em: 06 mai 2015]. 28 p. Contrato No.: GE.1410537. Report No.: 1410537. Disponível em: < http://www.srfood.org/images/stories/pdf/officialrepor ts/20140310_finalreport_en.pdf $>$

[2] Albuquerque MFM. A segurança alimentar e nutricional e o uso da abordagem de direitos humanos no desenho das políticas públicas para combater a fome e a pobreza. Rev nutr [Internet]. 2009 [Acesso em: 10 mai 2015]; 22(6):895-903. Disponível em: < http://dx.doi.org/10.1590/S1415-52732009000600011>

[3] Campello T, Neri MC, organizadores. Programa Bolsa Família: uma década de inclusão e cidadania [Internet]. Brasília: IPEA; 2013 [Acesso em: 10 mai 2015]. 494 p. Disponível em: $<$ http://www.ipea.gov.br/portal/index.php?option=com _content\&view $=$ article\&id $=20408>$

[4] Food and Agriculture Organization of the United Nations. O estado da segurança alimentar e nutricional no Brasil: um retrato multidimensional [Internet]. Brasília: FAO; 2014 [Acesso em: 10 mai 2015]. 87 p. Disponível em: <https://www.fao.org.br/download/SOFI_p.pdf>

[5] Ação Brasileira pela Nutrição e Direitos Humanos. Direito humano à alimentação adequada no contexto da segurança alimentar e nutricional [Internet]. Brasília: ABRANDH; 2010 [Acesso em: 10 mai 2015]. 204 p. Disponível em: <http://www.redsancplp.org/uploads/5/6/8/7/5687387/dhaa_no_contexto _da_san.pdf>

[6] BRASIL. Ministério da Saúde. Portaria 2.446, de 11 de novembro de 2014. Redefine a Política Nacional de Promoção da Saúde (PNPS).

[7] Casemiro JP, Valla VV, Guimarães MBL. Direito humano à alimentação adequada: um olhar urbano. Cienc. saude colet [Internet]. 2010 [Acesso em: 20 jun 2015];15(4):2085-2093. Disponível em: $<$ http://dx.doi.org/10.1590/S1413$81232010000400022>$

[8] BRASIL. Lei 10.836, de 09 de janeiro de 2004. Cria o Programa Bolsa Família e dá outras providências. Brasília: Ministério da Saúde; 2004.

[9] Ministério do Desenvolvimento Social e Combate à Fome. Bolsa Família [Internet]. Brasília: Governo Federal do Brasil; [Acesso em: 08 mai 2015]. Disponível em: $<$ http://mds.gov.br/assuntos/bolsa-familia $>$

[10] Trevisani JJD, Burlandy L, Jaime PC. Fluxos decisórios na formulação das condicionalidades de saúde do programa bolsa família. Saúde Soc [Internet]. 2012 [Acesso em: 11 mai 2015]; 21(2):492-509. Disponível em: <http://dx.doi.org/10.1590/S0104-

$12902012000200021>$. 
[11] Ministério da Saúde; Secretaria de Atenção à Saúde; Departamento de Atenção Básica. Guia alimentar para a população brasileira: promovendo a alimentação saudável [Internet]. Brasília: Ministério da Saúde; 2008 [Acesso em: 12 mai 2015]. 210 p. (Série A. Normas e Manuais Técnicos). Disponível em: <http://bvsms.saude.gov.br/bvs/publicacoes/guia_alime ntar_populacao_brasileira_2008.pdf>

[12] Ministério da Saúde; Secretaria de Atenção à Saúde; Departamento de Atenção Básica. Guia alimentar para a população brasileira [Internet]. 3 ed. Brasília: Ministério da Saúde; 2014 [Acesso em: 10 mai 2015]. 152 p. Disponível em: <http://portalsaude.saude.gov.br/images/pdf/2014/nov embro/05/Guia-Alimentar-para-a-pop-brasiliera-MioloPDF-Internet.pdf $>$

[13] Redenutri: rede de alimentação e nutrição do sistema único de saúde [Internet]. Brasília: Ministério da Saúde. 2011. Ação Brasileira para a Nutrição e Direitos Humanos. Peraí, é nosso direito!; [Acesso em: 08 mai 2015]. Disponível em: <http://ecos-redenutri.bvs.br/tikiindex.php?page $=$ video_peraienossodireito $>$

[14] Deus GI, Garcia SAVM. Capacitação em técnicas culinárias. Goiânia: Faculdade de Nutrição/UFG; 2010. 20 pág. (Texto produzido para uso nas atividades do projeto])

[15] Monego ET, Cunha DTO, Gil MF, et al. Alimentação saudável e segura em casa é um direito! Goiânia: Secretaria de Estado da Cidadania e Trabalho; 2009. 48 pág (Cartilha impressa para uso nas atividades do projeto)

[16] Ferreira VA, Magalhães R. Nutrição e promoção da saúde: perspectivas atuais. Cad Saúde Pública [Internet]. 2007 [Acesso em: 11 mai 2015]; 23(7):1674-81. Disponível em: <http://www.scielo.br/scielo.php?script=sci_arttext\&pid $=$ S0102-311X2007000700019>

[17] Rückert G, Gaia MCM. Educação popular, saúde e segurança alimentar em áreas do movimento dos trabalhadores rurais sem terra. Tempus Actas Saúde Colet [nternet]. 2014 [Acesso em: 10 mai 2015]; 8(2):157-71. Disponível <http://dx.doi.org/10.18569/tempus.v8i2.1517>

[18] Paes-Sousa R, Santos LMP, Miazaki ES. Effects of a conditional cash transfer programme on child nutrition in Brazil. Bull World Health Org [Internet]. 2011 [Acesso em: 11 mai 2015];89:496-503. Disponível em: <http://www.who.int/bulletin/volumes/89/7/10084202.pdf $>$

[19] Rasella D, Aquino T, Santos CAT, et al. Effect os a conditionalcash transfer programe on childhood mortality: a Nationwide analysis of Brazilian municipalities. Lancet. 2013; 382(9886):57-64.
[20] Burlandy L. Transferência condicionada de renda e segurança alimentar e nutricional. Ciênc Saúde Colet [Internet]. 2007 [Acesso em: 11 mai 2015]; 12(6):1441-51. Disponível em: <http://dx.doi.org/10.1590/S141381232007000600007>

[21] Cotta RMM, Machado JC. Programa Bolsa Família e segurança alimentar e nutricional no Brasil: revisão crítica da literatura. Rev. Panam. Salud Pública [Internet]. 2013 [Acesso em: 11 mai 2015]; 33(1):54-60. Disponível em: $<$ http://dx.doi.org/10.1590/S1020 49892013000100008>

[22] Magalhães R. Avaliação de políticas e iniciativas públicas de segurança alimentar e nutricional: dilemas e perspectivas metodológicas. Ciênc Saúde Colet [Internet]. 2014 [Acesso em: 11 mai 2015]; 19(5):1339-46. Disponível em: <http://dx.doi.org/10.1590/141381232014195.12202013>

Agradecimentos:

Demais participantes deste projeto: Dulce $\mathrm{T}$. O. Cunha, Gilciléia I. Jesus, Maria de Fátima Gil, Sílvia Aguiar V.M. Garcia, Simoni U. Silva, Valéria de S. Abreu e a equipe técnica da SECT-GO, Esther D. Santos (in memorian) e Selma F. S. Bernardes. 